\title{
A ratio principle for a red/green and a yellow/blue channel?
}

\author{
KARL-HEINZ BÄUML \\ Universität Regensburg, Regensburg, Germany
}

\begin{abstract}
There is strong empirical evidence that, under adaptation to another achromatic color stimulus, the lightness of an achromatic color stimulus depends on the ratio of the luminances of the two stimuli. In the present study, the suitability of this ratio principle is tested for two chromatic postreceptoral opponent channels. A Hering red/green channel and a non-Hering yellow/blue channel are specified as chromatic channels. The yellow/blue channel is defined by extrapolating the plane corresponding to unique green-white linearly to the reddish part of color space, using the plane's surface as the channel's equilibria. The experiment was run on an isoluminant plane, measured individually for each observer. Moving along an observer's measured opponent axes, eight adaptation stimuli were selected for each channel and spanned the whole range of the channel's coordinates. Red/green equilibria or yellow/blue equilibria were measured as excursions along the adaptation axes. For both presumed channels, the ratios of the equilibrium coordinates of test and adaptation stimuli were essentially constant. This supports the principle's suitability. However, small asymmetries were found with respect to each channel's opponent hues. The status of the proposed yellow/blue channel is discussed, as are conditions that might have favored the present findings.
\end{abstract}

Wallach (1948) postulated that, under adaptation to another achromatic stimulus, the lightness of an achromatic test stimulus depends on the ratio of the luminances of the two stimuli. The validity of this ratio principle has been the issue in many experimental studies using both temporal and surrounding adaptation conditions (for a review, see Jacobsen \& Gilchrist, 1988). Especially because of the extensive work of Jacobsen and Gilchrist (1988), there is now strong evidence that this principle really holds, at least in decremental stimulus configurations-that is, when the luminance of the test stimulus does not exceed that of the adaptation stimulus. The effect of changes in adaptation level on the lightness of a test stimulus can therefore be described in a very simple way.

Some theories of color adaptation have implicitly assumed the ratio principle to hold for the chromatic case as well. For instance, one line of reasoning argues that von-Kries-type transformations underlie color appearance changes. Here, the effect of an adapting stimulus on the appearance of a test stimulus has sometimes been supposed to be governed by the ratios between the color coordinates of the two stimuli, determined separately for each cone type (von Kries, 1905; Land, 1977). However, even if the assumption that the ratio principle holds for each cone type separately is dropped, there are no data to support the principle in the chromatic case (Wyszecki \& Stiles, 1982).

Correspondence should be addressed to the author at the Institut für Psychologie, Universität Regensburg, 8400 Regensburg, Germany (email: heinz@rpss3.psychologie.uni-regensburg.de)
Most modern theories of color appearance presume three postreceptoral channels-two chromatic channels and an achromatic one-which combine the outputs from the three cone types. The chromatic channels are usually supposed as an opponent red/green channel and an opponent yellow/blue channel; the achromatic channel is usually identified with luminance (Wyszecki, 1986; Wyszecki \& Stiles, 1982). With this postreceptoral stage in mind, the experimental situation in the studies of Jacobsen and Gilchrist (1988) and of others using achromatic stimuli can be characterized by a control, or zero coordination, of the two chromatic channels, whereas the achromatic channel is systematically varied. Jacobsen and Gilchrist found the ratio principle to be true for the achromatic channel. Following this approach, a generalization of the principle to chromatic postreceptoral opponent channels may be supposed. Similar to the achromatic case, for each chromatic channel, a control, or zero coordination, of the other opponent channels-that is, the other chromatic channel and the achromatic channel-is assumed. Over and above these presumably crucial side conditions, the stimulus configuration is restricted in order to be decremental-that is, the opponent coordinate of the test stimulus is not allowed to exceed that of the adaptation stimulus. Applied to, say, a red/green channel, this interpretation of the ratio principle predicts a red/green equilibrium configuration - that is, a test stimulus appearing unique yellow, unique blue, or achromatic under some adaptation stimulus-to remain in equilibrium if the ratio of the red/green coordinates of the two stimuli is not changed. All stimuli are presupposed to have identical luminances and to be zero-coordinated for the second chro- 
matic channel. The application of the ratio principle to a yellow/blue channel is similar.

This interpretation of the ratio principle has not been explicitly tested before. However, some published data provide a test of this principle under less restrictive side conditions than those formulated above. Data from Walraven (1976) and Shevell (1978) show the principle to fail for the red/green channel, at least when the other channels are not controlled. They used yellowish red adaptation stimuli with nonzero yellow/blue coordinates. In addition, the luminances of the test and adaptation stimuli were not identical. For the same chromatic channel, data from Cicerone, Krantz, and Larimer (1975) suggest that the principle does not even hold for adaptation stimuli that are zero-coordinated with respect to the yellow/blue channel, at least not when test and adaptation stimuli differ in luminance. The goal of the present study is to examine the validity of the principle's more restrictive interpretation for two chromatic postreceptoral channels.

The study is based on the specification of three linear axes in color space. Besides an achromatic channelluminance-a red/green channel and a yellow/blue channel are specified. The definition of the red/green channel is founded on the well-established finding that the red/green equilibria-namely, unique yellow and unique blue-fall on the surface of a plane in color space (Larimer, Krantz, \& Cicerone, 1974). The same is not true for the equilibria of a Hering yellow/blue channel. The empirical conditions that have to be fulfilled for these equilibria to fall on the surface of a plane-namely, additive and scalar closure (Krantz, 1975)-were found to be clearly violated (Elzinga \& deWeert, 1984; Larimer, Krantz, \& Cicerone, 1975; Suppes, Krantz, Luce, \& Tversky, 1989). Thus, a different second postreceptoral opponent channel is chosen. On the basis of unique green's Abney hue invariance and Bezold-Brücke invariance (Burns, Elsner, Pokorny, \& Smith, 1984; Larimer et al., 1975; Purdy, 1931), and of Werner and Walraven's (1982) finding that the achromatic locus falls on a straight line in color space, a plane in color space is defined by extrapolating the plane unique green-white linearly to the reddish part of color space. The resulting plane's surface in color space is taken to define the equilibria for a linear yellow/blue channel. There is no guarantee that this plane's surface can be specified in terms of only two hues, as is true for the red/green channel. But, to avoid circumlocution, I use the word "pink" to refer to the nongreen hue(s). Notice that the empirical soundness of this phenomenal simplification critically depends on Abney hue invariance and Bezold-Brücke invariance for pink to hold (see Discussion).

On the basis of this opponent-colors framework, an experiment is reported that examines the ratio principle for the two supposed chromatic channels. On a fixed isoluminant plane, eight adaptation stimuli are selected for each channel that spanned the whole range of the respective opponent channel. Four pink (unique yellow) and four unique green (unique blue) adaptation stimuli are used, starting with the highest red (yellow) coordinate and ending at the highest green (blue) coordinate. The test stimuli presented are excursions along the adaptation axes. For each channel, equilibria are measured under all eight adaptation conditions. The ratio principle predicts that the equilibria will share a constant ratio of the opponent coordinates for test and adaptation stimuli.

\section{METHOD}

\section{Observers}

A total of five naive psychology students and the author served as observers-three observers for each opponent channel. All observers had normal color vision.

\section{Stimuli}

The stimuli were displayed on a color monitor (BARCO CDCT $51 / 3)$, with a refresh rate of $50 \mathrm{~Hz}$ in noninterlaced video mode. The red, green, and blue channels of the monitor were controlled by an 8-bit digital-to-analog converter. The signals of the color channels could be varied in 256 steps from zero to maximal intensity for each pixel. There was a software control of the monitor's input signal correcting local variations of the monitor's energy output and nonlinearities in the tube's response function. The luminance measurements of the single color channels were based on the manufacturer's C.I.E. phosphor coordinates. Luminance was measured at eye position with the use of a photometer with very precise $V(\lambda)$ calibration (LMT 1000, Lichtmesstechnik, Berlin).

\section{Measuring the Individual Isoluminant Planes}

C.I.E. luminance is not a very perfect measurement of sensation luminance (Kaiser, 1988); therefore, as a result of using C.I.E. luminance as a code for sensation luminance, the sensation luminance of short-wave color stimuli is often underestimated. Furthermore, sensation luminance is subject to considerable individual variations (LeGrand, 1968). This motivates a better measure of sensation luminance. C.I.E. luminance, therefore, was corrected by measuring individual planes of constant sensation luminance. Taking linearity of sensation luminance for granted (Yellot, Wandell, \& Cornsweet, 1984) a plane of constant sensation luminance can be determined from knowing the C.I.E. luminance values of just three color stimuli with the same sensation luminance.

A flicker fusion experiment was conducted. The point $x=y=1 / 3$ in the C.I.E. diagram was used as the standard stimulus, with a C.I.E. luminance of $25 \mathrm{~cd} / \mathrm{m}^{2}$. The three monitor primaries served as comparison stimuli. The field was a disk with $6^{\circ}$ in visual angle. The stimuli were presented steadily. The situation was monocular. The settings were preceded by 7 min of dark adaptation. By using a method of adjustment, the observer's task was to vary the C.I.E. luminances of the primaries until flicker was just detected. The method of adjustment that was used had four settings for each comparison stimulus. As in the two experiments reported below, the programming was done by using PXL subroutines (Irtel, 1992).

The results, in fact, showed some variations across observers. However, all observers consistently adjusted higher C.I.E. luminances for monitor green and monitor red, in contrast to the adjustments for monitor blue. Also, for all observers, the C.I.E. luminance values for monitor green were a bit higher than those for monitor red. These results are consistent with the 1931 C.I.E. Standard Observer (see also LeGrand, 1968). The measured C.I.E. luminance values were taken for each observer to define his or her individual plane of constant sensation luminance. These planes were used for the rest of the study, so that all color stimuli presented to an observer lay on his or her individual isoluminant plane. 


\section{Measuring the Individual Opponent Axes}

A test of the ratio principle for the two chromatic channels requires measuring the unique yellow-unique blue axis and the unique green-pink axis for each observer's isoluminant plane. In fact, several studies show that the location of these axes can vary strongly across observers (cf. Larimer et al., 1974, 1975).

Procedure. The unique yellow-unique blue axis for the presumed $\mathrm{red} / \mathrm{green}$ channel was measured. This was done by measuring four to eight red/green equilibria-that is, colors that appeared neither reddish nor greenish but varied with respect to their yellowness or bluishness-for each observer. On the basis of these measurements, a straight line unique yellow-unique blue was fitted for each observer. For the presumed yellow/blue channel, unique green was measured. This was done by measuring for each observer four colors that appeared neither yellowish nor bluish but varied with respect to their greenness. On the basis of these measurements, a straight line unique green-white was fitted for each observer and linearly extrapolated to the reddish part of the diagram.

The experiment was programmed by using polar coordinates with zero point $x=y=1 / 3$ within the C.I.E. diagram. In these coordinates, the angle approximates hue, and the distance from zero point approximates saturation. For each hue-yellow, green, and blue-four saturation levels were defined as circles around the zero point, with different distances from it. For each hue and each saturation level, only stimuli falling on this circle were presented. The observer's task was to judge whether the stimulus was more reddish or more greenish, or more yellowish or more bluish. The sequence of stimulus presentations was determined by an adaptive procedure combining a stochastic approximation and a staircase method (Falmagne, 1985). Four staircases were always run simultaneously, switching irregularly among each other. For each hue and each saturation level, at least six adaptive procedures were run.

Each experimental session began with 7 min of dark adaptation. Stimuli were exposed for $1 \mathrm{sec}$, with an intertrial interval of $12 \mathrm{sec}$ of darkness. There was no fixation point, so at the beginning of the 1 -sec exposure, the observer had to make an eye movement to tixate the circle. An acoustical signal preceded the presentation of a test stimulus by $3 \mathrm{sec}$. The monocularly seen field of view consisted of a circle subtending a visual angle of $2^{\circ}$. Except for this circle, the field of view was dark.

Results. For all observers, the three hues were Abney hue invariant. In addition, the unique yellow and unique blue colors were shown to be well-fitted by a straight line in the C.I.E. diagram (Figure 1). These results do not provide a very strong test of channel linearity. However, they are consistent with those reported in previous studies (see Larimer et al., 1974, 1975; Suppes et al., 1989).

\section{Chromatic Adaptation Sessions}

Scaling of axes. For each observer, the measured chromatic opponent axes were used to define two linear opponent channels. The axes were scaled so that the most saturated pink received red/green coordinate $\phi_{1}=1$, whereas the most saturated yellow received yellow/blue coordinate $\phi_{2}=1$. This implied negative $\phi_{1}$ values for green and negative $\phi_{2}$ values for blue. The achromatic point, correspondingly, had coordinates $(0,0)$.

Adaptation stimuli. For the defined red/green channel, the observers were adapted to four pink and four unique green color stimuli. Thus, in adapting to these stimuli, only the observer's supposed $\mathrm{red} / \mathrm{green}$ channel was varied, whereas his or her supposed yellow/ blue channel was zero coordinated. Pink adaptation stimuli were selected by first taking the pink color stimulus with the highest $\phi_{1}$ coordinate $\left(\phi_{1}=1\right)$ and then taking pink color stimuli with threefourths, two-fourths, and one-fourth parts of the coordinate, respectively $\left(\phi_{1}=.75, \phi_{1}=.5, \phi_{1}=.25\right)$. Green adaptation stimuli were selected in the same manner. First, the green stimulus with the highest negative $\phi_{1}$ coordinate was chosen, followed by green

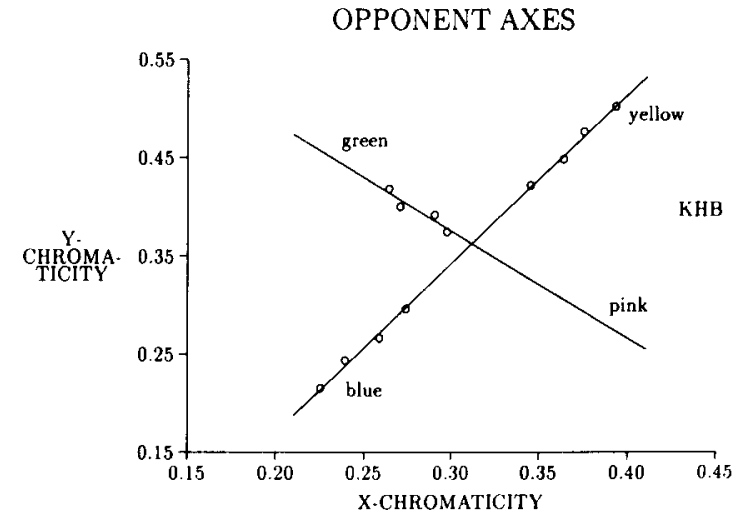

Figure 1. Estimated coordinates for unique blue, unique green, and unique yellow in C.I.E. diagram (Observer K.-H.B.). The coordinates for blue and yellow were fitted by a straight line. This line was used to define the observer's presumed red/green channel. The coordinates for green were also fitted by a straight line. This line then was used to define the observer's presumed yellow/blue channel (see text).

color stimuli with three-fourths, two-fourths, and one-fourth parts of the coordinate, respectively. The adaptation stimuli, therefore, spanned the whole range of the observer's red/green channel for the chosen isoluminant plane.

For the defined yellow/blue channel, the observers were adapted to four unique yellow and four unique blue color stimuli. Thus, in adapting to these stimuli, only the observer's supposed yellow/ blue channel was varied, whereas the supposed red/green channel was zero coordinated. Yellow and blue adaptation stimuli were selected in a way similar to that reported above for the red/green channel. The adaptation stimuli, therefore, spanned the whole range of the observer's yellow/blue channel for the chosen isoluminant plane.

Procedure. For each chromatic channel, equilibria were measured for each adaptation stimulus. The test stimuli presented were excursions along the adaptation axis. Therefore, in regard to the supposed red/green channel, only test stimuli that varied with respect to their red/green coordinates were presented, but they were zerocoordinated with respect to the supposed yellow/blue channel. Regarding the supposed yellow/blue channel, only test stimuli that varied with respect to their yellow/blue coordinates were presented, but they were zero-coordinated with respect to the supposed red/ green channel. The observer's task was to judge whether the stimulus presented on a trial was more reddish or more greenish, or more yellowish or more bluish, respectively (see below). Again, Falmagne's (1985) adaptive procedure was used. Two staircases were always run simultaneously, switching irregularly among each other. At least four adaptive procedures were run for each adaptation stimulus.

Each experimental session began with $7 \mathrm{~min}$ of dark adaptation. Then the observer fixated an adapting disk for $7 \mathrm{~min}$. Subsequently, the adaptation stimulus disappeared for $1 \mathrm{sec}$, during which time the test stimulus was presented. The test stimulus terminated, and the adapting stimulus immediately reappeared for $20 \mathrm{sec}$. The experiment continued with a $21-\mathrm{sec}$ cycle, consisting of $20 \mathrm{sec}$ of readapting exposure followed by $1 \mathrm{sec}$ of test exposure. Again, there was no fixation point, so at the beginning of the $1-\mathrm{sec}$ exposure, the observer had to make an eye movement to fixate the circle. This procedure is identical to the one used by Cicerone et al. (1975). An acoustical signal preceded the presentation of the test stimulus by $3 \mathrm{sec}$. The test field was a disk with $2^{\circ}$ in visual angle. The adapting stimulus also was a disk, but with $6^{\circ}$ in visual angle. The situation was monocular. 
Equilibria. For the red/green channel, equilibria are color stimuli that may appear either yellowish or bluish but neither reddish nor greenish - that is, unique yellow, unique blue, or achromatic. For the yellow/blue channel, things are not quite as nice. Here, equilibria are color stimuli appearing unique green, pink, or achromatic. For green, this means color stimuli that appear greenish but not yellowish or bluish. For pink, no criterion in terms of a missing yellowness or blueness exists; moreover, there is considerable doubt that pink relates to a uniquely determined hue at all (see introduction). A simple criterion for the yellow/blue channel, therefore, exists only if the adapting stimulus does not induce some redness in the appearance of the test stimulus. There is evidence that such conditions are met in the present study. For several adaptation conditions, Werner and Walraven (1982) found the achromatic locus to lie on a straight line in color space connecting the neutral achromatic locus with the respective adaptation locus. This finding implies that the achromatic loci for adaptation to yellow and blue fall on the straight-line yellow-blue. In fact, preliminary studies did not show obvious deviations from Werner and Walraven's hypothesis. The yellow/blue channel's equilibria were therefore reduced to color stimuli that appeared neither yellowish nor bluish.

\section{RESULTS}

\section{Red/Green Channel}

Table 1 contains, for each of the three observers, the pairs of red/green coordinates for test and adaptation stimuli that appeared as red/green equilibria. All observers show essentially the same pattern of results. Notice that under neutral adaptation-that is, given an adapting stimulus with a red/green coordinate $\phi_{1}=0$, a test stimulus with a red/green coordinate $\phi_{1}=0$ would be in neutral equilibrium. Adaptation leads to strong changes in the equilibrium locus. The changes fulfill a few moderate expectations well-known from other studies: First, the change in equilibrium locus is in the direction of the adaptation stimulus locus; second, there is a monotone relationship between the coordinates of test stimulus and adaptation stimulus, both for green and for pink. These characteristics were consistent for all three observers.

Table 1

Red/Green Channel: Red/Green Coordinate Pairs of Test and Adaptation Stimuli With the Adapted Test Stimulus Appearing in Red/Green Equilibrium

\begin{tabular}{cccccc} 
& \multicolumn{2}{c}{ Pink } & & \multicolumn{2}{c}{ Green } \\
\cline { 2 - 4 } Observer & $\mathrm{A}$ & $\mathrm{T}$ & $\mathrm{A}$ & $\mathrm{T}$ \\
\hline R.A. & 1.000 & 0.907 & & -0.583 & -0.503 \\
& $\mathbf{0 . 7 5 0}$ & 0.661 & & -0.438 & -0.379 \\
& 0.500 & 0.445 & & -0.291 & -0.255 \\
M.K. & 0.250 & 0.226 & -0.146 & -0.120 \\
& 1.000 & 0.812 & & -0.707 & -0.604 \\
& 0.750 & 0.609 & & -0.531 & -0.439 \\
W.O. & 0.500 & 0.404 & & -0.354 & -0.296 \\
& 0.250 & 0.244 & -0.177 & -0.131 \\
& 1.000 & 0.797 & -0.536 & -0.450 \\
& 0.750 & 0.571 & -0.402 & -0.376 \\
& 0.500 & 0.378 & -0.268 & -0.261 \\
& 0.250 & 0.195 & -0.134 & -0.131 \\
\hline
\end{tabular}

Note $-\mathrm{A}=$ coordinate of the adaptation stimulus; $\mathrm{T}=$ coordinate of the test stimulus. The coordinates were estimated individually for each observer.

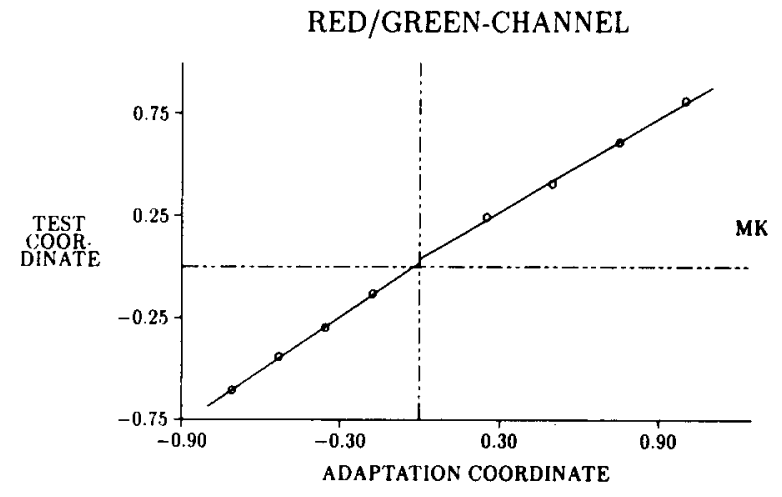

RED/GREEN-CHANNEL

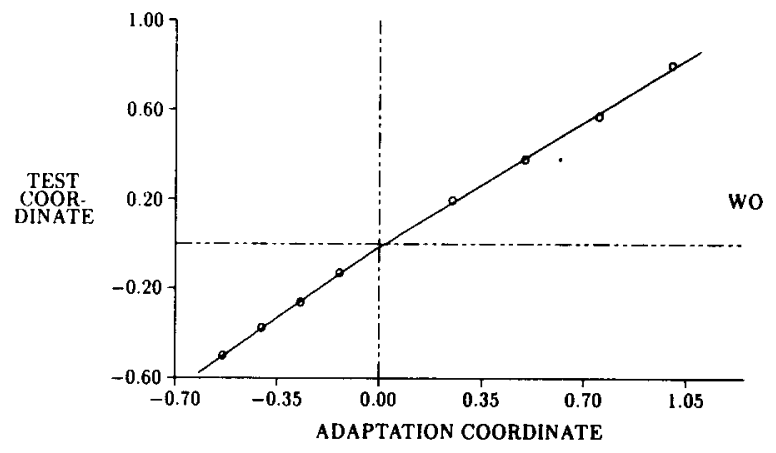

Figure 2. Red/green channel: Red/green coordinates of the equilibrium test stimuli as a function of the red/green coordinates of the adaptation stimuli, for adaptation to pink $\left(\phi_{1}>0\right)$ and to green ( $\phi_{1}<0$; Observers M.K. and W.O.).

Figure 2 shows, for two of the three observers, the $\mathrm{red} / \mathrm{green}$ coordinates of the test stimuli as a function of those of the adaptation stimuli. For the ratio principle to hold, the line connecting the various red/green coordinates of the test stimuli should be straight, intersecting the zero point of the coordinate system. The fitted straight lines reveal that this is true when the two hues are analyzed separately. The combination of the channel's two hues also fits a straight line quite well, with fairly small systematic deviations from linearity for two observers (M.K., W.O.), and essentially none for observer R.A.

\section{Yellow/Blue Channel}

Table 2 lists, for each of the three observers, the pairs of yellow/blue coordinates for test and adaptation stimuli that appeared as a yellow/blue equilibrium. All observers show essentially the same pattern of results. Under neutral adaptation, a test stimulus with yellow/blue coordinate $\phi_{2}=0$ would be in equilibrium. Again, adaptation induces strong changes in the equilibrium locus. As for the $\mathrm{red} / \mathrm{green}$ channel, the change in equilibrium locus is in the direction of the adaptation locus, with a monotone relationship between the coordinates of the test stimuli and the adaptation stimuli. This is true for yellow and for blue. 
Table 2

Yellow/Blue Channel: Yellow/Blue Coordinate Pairs of Test and Adaptation Stimuli With the Adapted Test Stimulus Appearing in Yellow/Blue Equilibrium

\begin{tabular}{ccccc} 
& \multicolumn{2}{c}{ Yellow } & \multicolumn{2}{c}{ Blue } \\
Observer & $\mathrm{A}$ & $\mathrm{T}$ & $\mathrm{A}$ & $\mathrm{T}$ \\
\hline K.-H.B. & $\mathbf{1 . 0 0 0}$ & 0.520 & -1.516 & -1.268 \\
& $\mathbf{0 . 7 5 0}$ & $\mathbf{0 . 3 8 5}$ & -1.137 & -0.953 \\
& $\mathbf{0 . 5 0 0}$ & $\mathbf{0 . 2 4 6}$ & -0.758 & -0.636 \\
F.G. & $\mathbf{0 . 2 5 0}$ & $\mathbf{0 . 1 1 2}$ & -0.379 & -0.315 \\
& $\mathbf{1 . 0 0 0}$ & 0.584 & -1.232 & -1.132 \\
& $\mathbf{0 . 7 5 0}$ & $\mathbf{0 . 4 0 1}$ & -0.924 & -0.848 \\
A.I. & $\mathbf{0 . 5 0 0}$ & $\mathbf{0 . 2 8 3}$ & -0.616 & -0.575 \\
& $\mathbf{0 . 2 5 0}$ & $\mathbf{0 . 0 9 6}$ & -0.308 & -0.346 \\
& 1.000 & 0.754 & -1.030 & -1.056 \\
& $\mathbf{0 . 7 5 0}$ & $\mathbf{0 . 6 0 4}$ & -0.772 & -0.826 \\
& $\mathbf{0 . 5 0 0}$ & $\mathbf{0 . 3 5 4}$ & -0.515 & -0.562 \\
& $\mathbf{0 . 2 5 0}$ & $\mathbf{0 . 1 5 1}$ & -0.257 & -0.342 \\
\hline
\end{tabular}

Note-A $=$ coordinate of the adaptation stimulus; $\mathrm{T}=$ coordinate of the test stimulus. The coordinates were estimated individually for each observer.

Figure 3 shows, for two of the three observers, the yellow/blue coordinates of the test stimuli as a function of those of the adaptation stimuli. The ratio principle predicts that the various yellow/blue coordinates of the test stimuli will fall on a straight line intersecting the zero point of the coordinate system. No observer's data differed from the principle's prediction, at least not when the two hues were regarded separately. The fitting of the coordinates for the two hues with only one straight line led to systematic deviations from linearity. These deviations were fairly small for observer A.I., but were more clear for the other two observers (K.-H.B., F.G.).

\section{DISCUSSION}

The present data support the suitability of the ratio principle for two supposed opponent channels, a red/green channel and a yellow/blue channel. For each channel, equilibrium configurations are shared by pairs of test and adaptation stimuli that show a constant ratio with respect to their channel's coordinates. The present data thus generalize the findings in the achromatic case (Jacobsen \& Gilchrist, 1988) to the chromatic case. The validity of the ratio principle for achromatic stimuli is critically tied to decremental stimulus configurations. Because of the employment of equilibrium configurations, the present study also used decremental configurations. This is again consistent with the findings in the achromatic case.

For the two hues of each channel, the deviations from the ratio principle were extremely small. The lines connecting the various opponent coordinates of the test stimuli are fairly linear and intersect the zero point of the coordinate system quite well. However, combining the two hues' coordinates for each channel led to some systematic deviations from linearity, suggesting different slopes for the two hues. This was most obvious for the yellow/ blue channel, whereas the deviations for the red/green channel were rather small. Interestingly, quite similar asymmetries were found in color discrimination experiments using the MacLeod-Boynton chromatic axes (Krauskopf \& Gegenfurtner, 1992). Notice, however, that the present results are based on the measurement of individual isoluminant planes and on the measurement of individual opponent axes. Failures in the supposed control of sensation luminance and the other chromatic channel might affect the outcome. Although the ratio principle implies constant coordinate ratios for equilibrium configurations for each single level of sensation luminance, it does not imply an invariance of the equilibrium locus with changes in the level of sensation luminance. Hence, systematic errors in estimating the isoluminant plane might have led to different slopes.

Two points regarding the power of the present test of the ratio principle should be stressed. First, the ratio principle was examined at a luminance level of about $25 \mathrm{~cd} / \mathrm{m}^{2}$. At this luminance level, the whole range of opponent coordinates was used for adaptation. Of course, by using a higher luminance level, more extreme opponent stimulations would have resulted, allowing more extended tests of the principle. Notice, however, that the luminance level employed is in the range of levels used
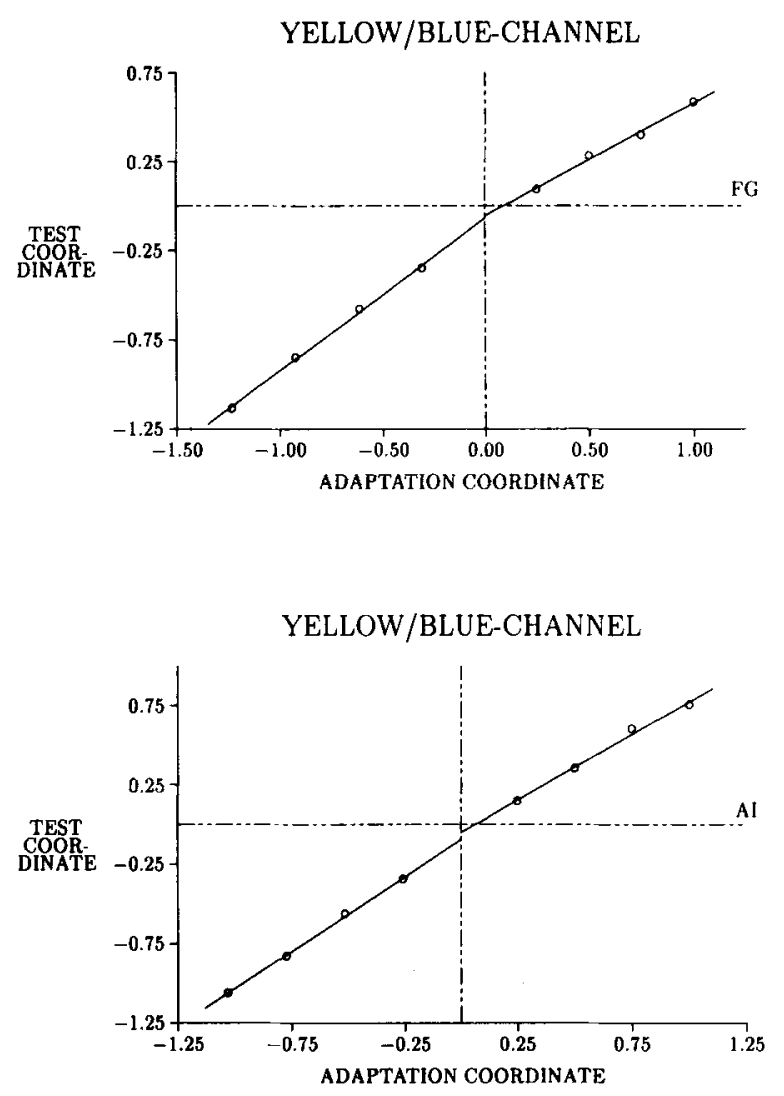

Figure 3. Yellow/blue channel: Yellow/blue coordinates of the equilibrium test stimuli as a function of the yellow/blue coordinates of the adaptation stimuli, for adaptation to yellow $\left(\phi_{2}>0\right)$ and to blue ( $\phi_{1}<0$; Observers F.G. and A.I.). 
in a number of other studies (Cicerone et al., 1975; Werner \& Walraven, 1982) that held local adaptations small; thus, it appeared to be an adequate starting point for testing. Second, the present study used equilibrium configurations to test the principle. Because of the strong adaptation effects, this use of equilibrium configurations puts considerable constraints on the possible coordinate ratios between test and adaptation stimuli. In fact, the ratio of the two opponent coordinates is never smaller than $1: 2$; more often it is in the range of 7:10 to $9: 10$ (see Tables 1 and 2). This limited range of coordinate ratios might have favored the validity of the principle.

In the chromatic adaptation sessions, the achromatic locus was assumed to fall on a straight line in color space connecting the neutral achromatic locus with the respective adaptation locus. This is what Werner and Walraven (1982) hypothesized and found empirical evidence for. In fact, our observers did not report any systematic deviations from this finding. Nonetheless, there is empirical evidence that Werner and Walraven's hypothesis is at best a simplification. Cicerone et al. (1975) found a linear red/green channel for adaptation to unique yellow and unique blue, with blue changing its spectral locus under adaptation to yellow, and yellow changing its spectral locus under adaptation to blue. Relying on this finding implies that the achromatic locus cannot fall on the straightline yellow-blue, which challenges Werner and Walraven's (1982) hypothesis. In the present study, however, the achromatic locus usually came close to the adaptation locus. Conceivable failures of Werner and Walraven's hypothesis, therefore, were not very critical.

The present results, combined with those in the achromatic case, motivate the definition of a postreceptoral stage that linearly combines the three receptor outputs. This is nothing new for the presumed red/green channel. Its status as a linear combination of the three receptor outputs is well-founded today (Suppes et al., 1989); in fact, data from one of the present preliminary experiments agree with this view. The same is not true, however, for the second opponent channel employed, the presumed yellow/blue channel. Its theoretical significance depends strongly on its phenomenal interpretability. This stresses the question of whether the channel's equilibria can be specified in terms of only two hues, unique green and pink. Although the greenish part does not appear problematic in this regard, the pinkish part indeed does. The channel's theoretical significance, therefore, critically relies on the validity of Abney hue invariance and BezoldBrücke invariance for "pink." 'Despite the considerable experimental work concerning hue invariances (see Wyszecki \& Stiles, 1982), it seems doubtful to me that this question has already been tested sufficiently. Instead, notice that there might be some relationship between the present pink and Purdy's (1931) fourth nonspectral Bezold-Brücke invariant hue. The question of whether the supposed yellow/blue channel really serves its own phenomenal status, or whether it only works as a linear ap- proximation of the well-known nonlinear Hering yellow/blue channel, is not yet solved.

In summary, the present findings for a red/green channel and a yellow/blue channel, together with those of Jacobsen and Gilchrist (1988) for an achromatic channel, suggest a ratio principle for three postreceptoral channels. For each of the three channels, however, there is only empirical support for the principle when the other two channels are controlled. Thus, whether the ratio principle will also hold for the achromatic channel in situations in which the two chromatic channels are not zerocoordinated is at the moment an open question. Similarly, it remains to be shown whether the ratio principle will also hold for the chromatic channels in situations in which the other two respective channels are not controlled, or zero-coordinated.

\section{REFERENCES}

Burns, S. A., Elsner, A. E., Pokorny, J., \& Smith, V. C. (1984) The Abney effect: Chromaticity coordinates of unique and other constant hues. Vision Research, 24, 479-489.

Cicerone, C. M., Krantz, D. H., \& Larimer, J. (1975). Opponentprocess additivity: III. Effect of moderate chromatic adaptation. $V i$ sion Research, 15, 1125-1135.

Elzinga, C. E., \&EWEert, C. M. M. (1984). Nonlinear codes for the yellow-blue mechanism. Vision Research, 24, 911-922.

FAlmaGne, J.-C. (1985). Elements of psychophysical theory. New York: Oxford University Press.

Irtel, H. (1992). PXL: A library for programming psychological experiments. Unpublished manuscript, Universität Regensburg, Regensburg, Germany.

JACOBSEN, A., \& GILCHRIST, A. (1988). The ratio principle holds over a million-to-one range of illumination. Perception \& Psychophysics, 43, 1-6.

KAISER, P. K. (1988). Sensation luminance: A new name to distinguish CIE luminance from luminance dependent on an individual's spectral sensitivity. Vision Research, 28, 455-456.

KrantZ, D. H. (1975). Color measurement and color theory: II. Opponent-colors theory. Joumal of Mathematical Psychology, 12, 304-327.

Krauskopf, J., \& Gegenfurtner, K. (1992). Color discrimination and adaptation. Vision Research, 32, 2165-2175.

LAND, E. H. (1977). The retinex theory of color vision. Scientific American, 237, 108-128

Larimer, J., Krantz, D. H., \& Cicerone, C. C. (1974). Opponent process additivity: I. Red/green equilibria. Vision Research, 14, $1127-1140$.

Larimer, J., Krantz, D. H., Cicerone, C. C. (1975). Opponent process additivity: II. Yellow/blue equilibria and nonlinear models. Vision Research, 15, 723-731.

LEGrand, Y. (1968). Light, colour and vision. London: Chapman \& Hall.

Purdy, D. M. (1931). Spectral hue as a function of intensity. American Joumal of Psychology, 49, 313-315.

SHEVELL, S. K. (1978). The dual role of chromatic backgrounds in color perception. Vision Research, 18, 1649-1661.

Suppes, P., Krantz, D. H., Luce, R. D., \& Tversky, A. (1989). Foundations of measurement (Vol. 3, pp. 226-298). San Diego: Academic Press.

voN KRJES, J. (1905). Die Gesichtsempfindungen. In W. Nagel (Ed.), Handbuch der Physiologie des Menschen (Vol. 3, pp. 109-279). Braunschweig, Germany: Vieweg.

WALLACH, H. (1948). Brightness constancy and the nature of achromatic stimuli. Journal of Experimental Psychology, 38, 310-324. 
Walraven, J. (1976). Discounting the background: The missing link in the explanation of chromatic adaptation. Vision Research, 16, 289-295.

WERNER, J., \& WALRAVEN, J. (1982). Effect of chromatic adaptation on the achromatic locus: The role of contrast, luminance and background color. Vision Research, 22, 929-943.

WYSZECKI, G. (1986). Color appearance. In K. R. Boff, L. Kaufman, J. P. Thomas (Eds.), Handbook of perception and human performance (Vol. 1, pp. 9-57). New York: Wiley.
Wyszecki, G., \& Stiles, W. S. (1982). Color science (2nd ed.). New York: Wiley.

Yellot, J. I., Wandell, B. A., \& Cornsweet, T. N. (1984). The beginning of visual perception: The retinal image and its initial coding. In I. Darian-Smith (Ed.), Handbook of physiology (Vol. 3, pp. 257-316). Bethesda, MD: American Physiological Society.

(Manuscript received January 22, 1992; revision accepted for publication August 5, 1992.)

\section{3rd Annual Meeting of the Society for Computers in Psychology Washington, D.C. November 4, 1993}

\section{Call for Papers}

The 23rd Annual Meeting of the Society for Computers in Psychology will be held at the Shoreham Hotel in Washington, DC, on November 4, 1993, the day before the annual meeting of the Psychonomic Society. The meeting will include presentations, workshops, tutorials, and demonstrations. The application of computerbased solutions to all areas of psychology will be featured, including research, education, clinical practice, and industrial applications. The proceedings will be published in Behavior Research Methods, Instruments, \& Computers.

The deadline for submissions is June 25, 1993.

For further information, contact Nancy Duncan, Department of Psychology, Hampton University, Hampton, VA $23668<71043,1340 @$ @compuserv.com $>$ or Ellen Rosen, Department of Psychology, College of William and Mary, Williamsburg, VA 23187 < Internet:EFROSE.MAIL.WM.EDU>. 